\title{
The Whole Mouse Brain: The Spatial Distribution and Morphology of Its Neurons
}

\author{
Bruce H. McCormick, ${ }^{*}$ David M. Mayerich, ${ }^{*}$ Brad L. Busse, ${ }^{*}$ Zeki Melek, ${ }^{*}$ Wonryull Koh, ${ }^{*}$ \\ Louise C. Abbott, ${ }^{* *}$ Yoonsuck Choe, ${ }^{*}$ John Keyser, ${ }^{*}$ Eun-Jung Kim* \\ *Dept. of Computer Science, Texas A\&M University, College Station, TX \\ **Dept. of Veterinary Integrated Biosciences, Texas A\&M University, College Station, TX
}

We are working to produce the first detailed map of 3D brain microstructure across a whole mammalian brain, that of the C57BL/6J mouse. Two tissue stains are used in this analysis: Nissl stain allows us to plot the spatial distribution and morphology of all cell bodies; and Golgi-Cox stain is used to elucidate the morphology of selective cells; approximately $1 \%$ of neurons stain in the Golgi stain. Three-dimensional reconstruction of only selected regions of Golgi-stained microstructure has been attempted to date, in view of the computational burden entailed.

A unique instrument of our invention, the Knife-Edge Scanning Microscope (KESM) [1], scans each entire mouse brain at $300 \mathrm{~nm}$ sampling resolution and will create an aligned volume data set of $\sim 7$ terabytes (uncompressed) representing the tissue microstructure. New tissue preparation techniques have been developed for en bloc staining and embedding with Nissl and Golgi-Cox stained tissue. In addition, new 3D parallel image-processing algorithms, including our polymerization algorithm $[2,3,4]$, have been developed to geometrically reconstruct and visualize the cell bodies and dendritic and axonal arborization of the neurons. Lastly, an I/O-intensive cluster computer supports the endeavor.

The counts, spatial distribution, and morphology of cell bodies within the mouse brain are derived from Nissl-stained tissue visualized at a 300nm sample interval along all three axes. This resolution allows us to measure the position, orientation (if discernible), and morphology of cell bodies while avoiding many of the ambiguities of stereological measurements. Reconstruction of the individual cell bodies in three dimensions from these measurements is relatively simple. Some refinements are added to partition occasional clusters of touching cells into neighboring but isolated cells.

For Golgi-Cox scanned tissue, we consider a fiber as part of a neuronal arbor (axonal or dendritic) whose soma does not reside within the immediate finite element (FE) under consideration for reconstruction. It is helpful to set up conventions for characterizing such fibers. One such convention we introduced is to assign a virtual soma to each distinguishable fiber, so that the fibers can be individually labeled and treated as "pseudo-neurons" as ranging across multiple FEs. The virtual soma serves as a "foster parent" of the fiber. These virtual somata are aligned along a bounding surface of the cluster of adjacent FEs currently under reconstruction. When fibers have been traced back to their actual somata, the corresponding fibers within the FE are relabeled as neuron segments for further reconstruction. 

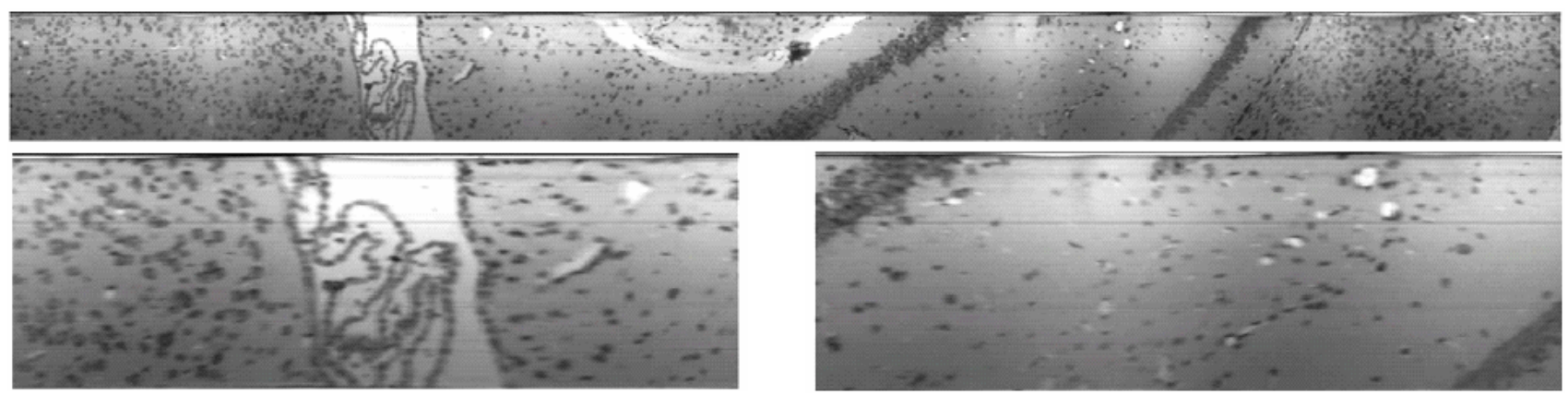

Fig. 1. KESM imaged strip of Nissl tissue (top) with close-ups to distinguish cell bodies (left, right).

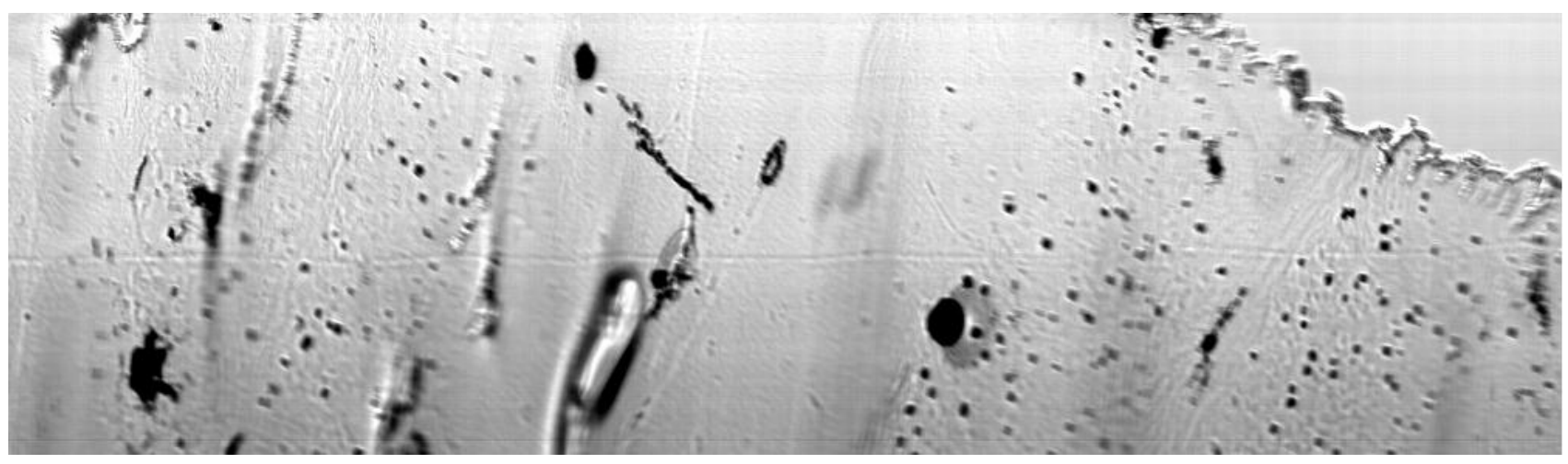

Fig 2. KESM imaged strip of Golgi tissue scanned at high magnification (300 $\mu \mathrm{m}$ sampling resolution).
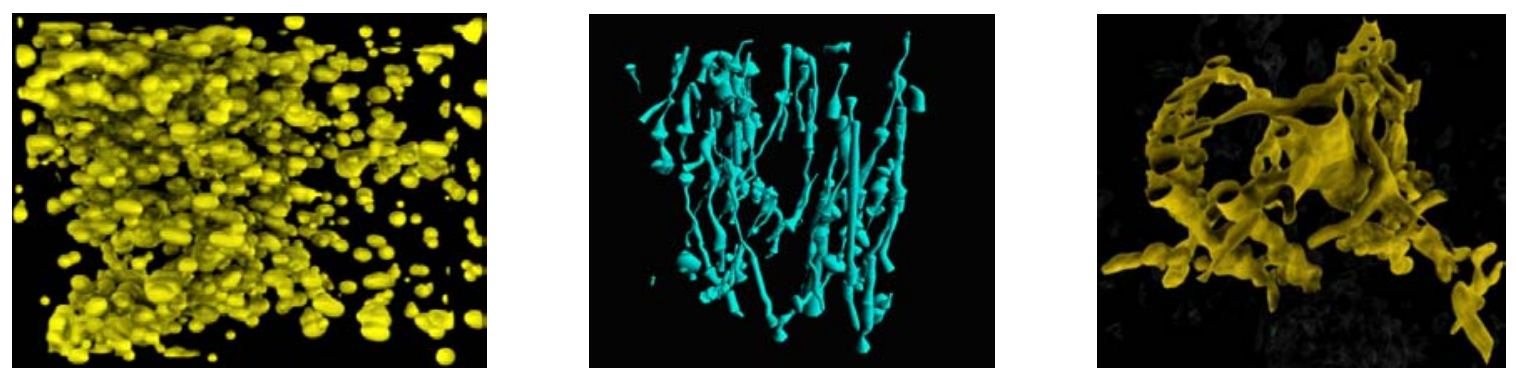

Fig 3. Sequential slices of Nissl tissue can be used to reconstruct cell positions and morphology (left). Neuronal fiber trajectories can be extracted from high-resolution sections of Golgi (center) and used to create visualizations of fiber tracts in conjunction with extracted radius information and isosurfaces for fibers and cell bodies (right).

[1] B.H. McCormick and D.M. Mayerich, Microsc. Microanal. 10, Suppl. 2, pp.1466-67, 2004.

[2] P. Aragonda, MS Thesis, Dep. Comp. Sci., TAMU, 2004

[3] P. Doddapaneni, MS Thesis, Dep. Comp. Sci., TAMU, 2004.

[4] B.H. McCormick et al., Proc. ACM Symp. Solid Modeling, pp. 333-38, June 2004.

[5] Support for the Brain Networks Laboratory contributed by: NSF-MRI Grant 0079874, Texas Higher Education Coordinating Board Grant ATP-00512-0146-2001 and the Office of the Vice President, Texas A\&M University. 